\title{
Multi-frame constrained block sparse Bayesian learning for flexible tactile sensing using electrical impedance tomography
}

This paper was downloaded from TechRxiv (https://www.techrxiv.org).

\section{LICENSE}

CC BY 4.0

SUBMISSION DATE / POSTED DATE

01-11-2021/09-11-2021

\section{CITATION}

Wang, Xiaojie (2021): Multi-frame constrained block sparse Bayesian learning for flexible tactile sensing using electrical impedance tomography. TechRxiv. Preprint. https://doi.org/10.36227/techrxiv.16910971.v1

$\mathrm{DOI}$

10.36227/techrxiv.16910971.v1 


\title{
Multi-frame constrained block sparse Bayesian learning for flexible tactile sensing using electrical impedance tomography
}

\author{
Gang $\mathrm{Ma}^{1}$, Haofeng Chen ${ }^{1,2}$, Peng Wang ${ }^{2}$ and Xiaojie Wang ${ }^{1,2}$
}

\begin{abstract}
In this paper, we presented a multi-frame constrained block sparse Bayesian learning (MFC-BSBL) reconstruction algorithm to tackle the challenge of poor-quality reconstruction images in electrical impedance tomography (EIT) for tactile sensing. The fundamental idea of MFC-BSBL is to explore the sparsity, intra-frame correlation, and inter-frame correlation of impedance distributions by extending the Bayesian inference framework. To verify the proposed algorithm, we conducted numerical simulations for different cases to identify one, multiple, round, and square targets. The simulation results demonstrated that this method can effectively detect the target positions and shapes by reducing artifacts and noise in the reconstructed images. To demonstrate the application of this approach to real EIT-based tactile sensing, we conducted real-contact detection experiments using the EIT tactile sensor system. Compared with traditional methods, the tactile sensor system using the MFCBSBL algorithm can achieve accurate contact detection and significantly reduce artifacts and noise.
\end{abstract}

Index Terms-Block Sparse Bayesian Learning, electrical impedance tomography, image reconstruction, tactile sensing.

\section{INTRODUCTION}

Recently, electrical impedance tomography (EIT) technology, as a special electrical detection method for nondestructive and radiation-free testing has been proposed for flexible tactile sensing [1], [2], [3], [4]. This technology constructs an image of the internal conductivity distribution by processing the potential measurement data from the boundary of the conductive material. The noninvasive imaging technology could realize a "one-piece" structure of a flexible tactile sensor without internal wires and units. The EIT-based flexible sensor can obtain the internal conductivity distribution of an electrically conductive body through its boundary electrodes. The position of the touching force can be estimated based on the conductivity distribution. EIT-based flexible sensors have advantages over conventional flexible sensors for simple structures and low cost.

A typical EIT-based tactile sensor usually consists of a rubberized and conductive material with multiple electrodes ( 8 or more) on its boundaries in response to a touch pressure with local changes in conductivity. The EIT is employed to reconstruct the contact region when the tactile sensor detects the pressure. However, mathematically, the EIT reconstruction

\footnotetext{
${ }^{1}$ University of Science and Technology of China, Hefei, Anhui 230026, China

${ }^{2}$ Institute of Advanced Manufacturing Technology, Hefei Institute of Physical science, Chinese Academy of Sciences, Changzhou 213164, China xjwang@iamt.ac.cn
}

problem is an ill-posed nonlinear inverse problem [5], [6], and the quality of the reconstructed images is limited by the reconstruction algorithms that are significantly affected by artifacts and noise. The poor quality reconstruction images would lead to inaccurate detection of the contact positions and contact forces.

Traditionally, the EIT tomography reconstruction inverse problem is solved by building a finite element (FE) model combined with regularization methods to reconstruct the internal conductivity of the object under detection. Reconstruction algorithms based on the Gauss-Newton (GN) algorithm with various priors such as Gaussian prior, Laplace prior and Newton's One-Step Error Reconstructor prior are common examples of FE techniques used to solve the EIT reconstruction inverse problems [7]. Although these methods provide good solutions for most EIT inverse problems, the reconstructed images suffer from low spatial resolution and are sensitive to measurement noise. Another approach uses the total variation (TV) regularization strategy to improve the reconstructed images. This method implemented the L1-norm regularization scheme to preserve the discontinuities of the boundaries and demonstrated an improved ability to reconstruct sharp contrasts [8]. However, the TV method causes undesired artifacts such as the staircase effect in reconstructing images.

In some typical cases of EIT image reconstruction, such as chest and lung imaging, the robustness and high spatial resolution solver of EIT can be achieved by optimizing the solution of the inverse problem with additional prior information in the solving process [9], [10], [11]. For example, Sun et al. [9] proposed an improved Tikhonov regularization method by obtaining a priori information of lung tissues to constrain the reconstructed images. Hamilton et al. [10] incorporated a priori knowledge of object boundaries to modify the D-bar method and adapted a convolutional neural network to acquire sharp and reliable reconstructions of EIT images. Liu et al. [11] proposed shape-driven reconstruction approaches by reformulating the EIT inverse problem as a shape-reconstruction problem and implemented more geometry and prior information into the solution process. However, the accuracy and efficiency of these methods in EIT image reconstruction depend heavily on extra prior information of objects of interest, such as shapes and relative positions. Additionally, many regularization parameters must be tuned accordingly, which makes them more difficult to be used in real applications.

Comparing with traditional electrical tomography ap- 
proaches which are based on the system's FE model to solve the EIT inverse nonlinear problem, a supervised machine learning (ML) method was proposed by Russo et al [2] to address the touch position issue of an EIT based flexible tactile sensor. They built a ML model to obtain the mapping relationship between the impedance change distribution and boundary measurement data. By training the model with a large amount of data, they can achieve a more accurate position and faster response in touch sensing. However, because the actual impedance change is extremely complicated and affected by the contact position, shape and target numbers, the ML model cannot establish a complete mapping relationship between the internal impedance distribution and the measurement data. The ML method can only be applied to some simple cases. Similarly, Park et al. [12] proposed adapting a deep neural network approach to an EIT-based tactile sensor. Zhang et al. [13] presented a textile-based tactile sensor using a neural network approach to detect the contact position. Wei et al. proposed a reliable deep learning scheme (RDLS) by incorporating the physical information along with Bayesian convolutional neural network to deal with typical nonlinear EIT reconstruction problems[14]. They achieved fast, highquality and robust images with pixel-based uncertainties in both simulation and experimental tests [14]. However, this method is sensitive to the physical information of the region of interest (ROI). The networks have to be re-trained if the ROI changes. All of these methods based on ML techniques are not universal, because each model can only be applied in either one-target contact or two-target contact condition.

Recently, block sparse Bayesian Learning (BSBL)[15] has attracted attention for solving the EIT inverse problem in medical images [16], [17]. BSBL has the characteristics of flexible modeling and strong robustness in noisy environments. It can adaptively explore and utilize the underlying data structure [15]. Liu et al proposed a structure-aware sparse Bayesian learning algorithm to improve the EIT reconstruction quality [16]. This method incorporates the structure-aware priors in a single-frame impedance distribution, and is capable of improving the image resolution and avoiding time-consuming parameter tuning processes. Later, they updated the learning rule by using the approximate messaging algorithm (AMP) method to accelerate the solution process in 3D cell pellet imaging [17]. Under certain assumptions, the AMP algorithm can simplify matrix calculations using approximations, thereby reducing the computational complexity. However, this method does not work for cases where the measurement matrix has highly correlated columns. Xiang et al. introduced a multi-frequency BSBL method in an electromagnetic tomography (mfEMT) system for the initial diagnosis of acute stroke [18]. Because the conductivity of brain tissues and strokes is a function of the excitation frequencies, by exploiting the frequency constraint information between the brain conductivity distributions at different excitation frequencies, they can improve the spatial resolution of reconstructed images to detect acute strokes in the brain region. Yin et al. adapted the BSBL method to bioluminescence tomography (BLT) for tumor morphology reconstruction [19]. By exploring the clustering characteristics of the points around the light source with similar intensities, they can achieve an accurate BLT reconstruction of tumor location and morphology. These studies illustrated that BSBL-based methods can be effectively used to solve inverse problems of medical images.

In this paper, we propose a reconstruction approach called multi-frame constrained block sparse Bayesian learning (MFC-BSBL) based on a linearized multi-frame model, and designed a tactile sensor system using MFC-BSBL to achieve accurate contact position detection and contact region detection. The fundamental idea of MFC-BSBL is to explore the correlation among images under a set of frames by extending the BSBL framework. Mathematically, such correlations can be described as a constrained optimization problem that promotes the block sparsity of the impedance distribution. We present a multi-frame constrained block sparsity prior that incorporates the sparsity, intra-frame correlation and interframe correlation of the conductivity distribution.

To evaluate the performance of the proposed MFC-BSBL reconstruction algorithm as well as the EIT tactile sensor system, we conducted numerical simulations for different cases to identify one, multiple, round, and square targets. The simulation results suggest that this method can effectively detect the contact region and the contact position. Additionally, it can reduce artifacts and noise in the reconstructed images. To demonstrate the application of this approach to real tactile sensing, we designed a portable EIT tactile sensor system and conducted real-contact detection experiments. Compared with traditional methods, the tactile sensor system using the MFCBSBL algorithm can achieve accurate contact detection and significantly reduce artifacts and noise.

The main contributions of this work are listed as follows:

- We proposed a MFC-BSBL reconstruction method that can significantly improve the poor-quality reconstruction images in EIT for tactile sensing. In addition, this method can realize the contact detections and localization of different shapes and multiple targets by adaptively learning the sparsity, intra-frame correlation, and inter-frame correlation priors of the conductivity distribution.

- We designed a portable EIT data acquisition system based on integrated circuit for tactile sensor. Example applications demonstrate that the EIT-based tactile sensor with MFC-BSBL owns the capabilities of removing artifacts and noise, and accurate contact position detection and contact region detection.

\section{Methodology}

\section{A. Forward problem}

The problem of EIT reconstruction refers to finding the distribution of impedance inside an object when a set of measured currents and voltages are known, which is a nonlinear and illposed inverse problem in which the mathematically obtained solutions are not necessarily unique.

For a bounded domain enclosed $\Omega$ by a boundary $\partial \Omega$, the forward problem is to calculate the voltages on the boundary of a tactile sensor through known impedance distribution and injected currents at the boundary. To derive the mathematical model of this physics problem, we adopt the complete 
electrode model (CEM) [20] and solve the Laplacian elliptic partial differential equation

$$
\nabla \cdot[\sigma(x, y) \nabla \phi(x, y)]=0,(x, y) \in \Omega
$$

with boundary conditions (Dirichlet and Neumann)

$$
\begin{gathered}
\int_{e_{l}} \sigma(x, y) \frac{\partial \phi(x, y)}{\partial n} d S=I_{l}, l=1, \ldots, D \\
\sigma(x, y) \frac{\partial \phi(x, y)}{\partial n}=-J_{s} \cdot n \equiv j,(x, y) \in \bigcup_{l=1}^{D} e_{l} \\
\sigma(x, y) \frac{\partial \phi(x, y)}{\partial n}=0,(x, y) \in \partial \Omega \backslash \bigcup_{l=1}^{D} e_{l}
\end{gathered}
$$

where $D$ is the number of electrodes, $j$ denotes the negative normal component of the injected density $J_{s}$ on the boundary, $n$ is an outward unit normal, $\sigma$ is the electrical conductivity, and $\phi$ is the electrical potential. To ensure the uniqueness of the solution, the conservation of charge theorem must hold

$$
\int_{\partial \Omega} j=0
$$

A commonly used method to solve the forward model (1-4) is the finite element method (FEM). The continuous form of the problem is transformed into a discrete approximation, which is constructed as a finite collection of $K$ elements with constant conductivity defined by $M$ interconnected nodes [21].

\section{B. Inverse problem}

Dynamic imaging or difference imaging is a fast method of imaging based on the difference between two measured states, which can readily reduce systematic artifacts through measurement cancelation. Hence, we adopted linearized dynamic imaging in our work. The essence of the method is to first calculate the boundary potentials $\mathbf{V}$ under an assumed homogenous domain with known conductivity $\sigma_{0}$. Then, a linear approximation is used to replace the discrete model to calculate the difference $\delta \sigma$, and the linearized problem becomes:

$$
\delta \mathbf{V} \approx \mathbf{J} \delta \sigma+\mathbf{N}
$$

where $\delta \mathbf{V} \in \mathbb{R}^{M \times L}$ is a matrix containing measurement voltages of $L$ frames, $\mathbf{N} \in \mathbb{R}^{M \times L}$ denotes the noise matrix, and $\mathbf{J} \in \mathbb{R}^{M \times N}$ is the Jacobian matrix that can be obtained by perturbing the elements of the FEM mesh $K$ and solving the forward problem of calculating the potential $\delta \mathbf{V}$

$$
\delta \mathbf{V}^{(d, m)}=-\iiint_{\Omega} \delta_{\gamma} \nabla u\left(I^{d}\right) \cdot \nabla u\left(I^{m}\right) d x d y d z
$$

where $\delta \mathbf{V}^{(d, m)}$ is the measurement $m$-th under the current $d$-th pattern. $u\left(I_{d}\right)$ and $u\left(I_{m}\right)$ denote the measured potential and current potentials, respectively. Taking $\delta_{\gamma}$ to be the $k$-th element, $J$ can be calculated as:

$$
\mathbf{J}_{(d, m ; k)}=\frac{\partial \mathbf{V}^{(d, m)}}{\partial \sigma_{k}}=-\iiint_{\Omega_{k}} \nabla u\left(I^{d}\right) \cdot \nabla u\left(I^{m}\right) d x d y d z
$$

For convenience of notation, $\delta \mathbf{V}$ and $\delta \sigma$ are simplified as $\mathbf{V}$ and $\sigma,(5)$ can be rewritten as

$$
\mathbf{V}=\mathbf{J} \sigma+\mathbf{N}
$$

where $\mathbf{V}=\left[v_{1}, v_{2}, \ldots v_{L}\right] \in \mathbb{R}^{M \times L}, \mathbf{J} \in \mathbb{R}^{M \times N}, \sigma=$ $\left[\sigma_{1}, \sigma_{2}, \ldots, \sigma_{L}\right] \in \mathbb{R}^{N \times L}, \mathbf{N}=\left[n_{1}, n_{2}, \ldots, n_{L}\right] \in \mathbb{R}^{M \times L}$.

Because the current passing through most elements is small, many Jacobian elements will have values close to zero. Dividing by such a small value will lead to numerical sensitivity in the solution so that small noises in the measured potentials will cause large errors in the reconstruction process. As such, regularization techniques are utilized to solve this illconditioned problem and stabilize the EIT solutions.

$$
\hat{\sigma}=\min _{\sigma} d(\mathbf{V}, \mathbf{J} \sigma)+\lambda R(\sigma)
$$

Where $d(\mathbf{V}, \mathbf{J} \sigma)$ denotes the data-fitting term that enforces $\sigma$ to satisfy the measured $\mathbf{V}$, and $R(\sigma)$ is a regularization term. Informally, regularization means that additional (prior) information is introduced such that an ill-posed problem can be replaced by a nearby well-posed problem, such as $\sigma$ is slowly changing, smooth, or blocky.

From a statistical perspective, the ill-posed problem of (9) can be reformulated to find the maximum a posteriori (MAP) of $\sigma$ given $\mathbf{V}$. According to Bayes' rule of probability, we have

$$
\hat{\sigma}=\underset{\sigma}{\arg \max } p(\sigma \mid \mathbf{V})=\underset{\sigma}{\arg \max } \log (p(\mathbf{V} \mid \sigma))+\log p(\sigma)
$$

where the log-likelihood term $p(\mathbf{V} \mid \sigma)$ enforces the data fidelity, and the prior term $p(\sigma)$ denotes the a priori knowledge (i.e., structural constraints) that stabilizes the inverse problem. Compared to conventional approaches, SBL assigns probabilities rather than deterministic values to model parameters by combining the data modal and a priori. The posterior probability of the model parameter conditioned on the observed data describes all possible solutions to the inverse problem along with their probabilities, which is essential for uncertainty quantification and makes it robust to noise disturbance.

\section{Reconstruction algorithm: MFC-BSBL method}

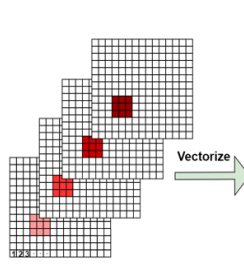

(a)
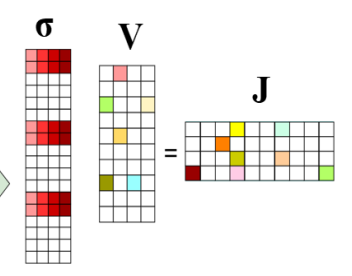

(b)

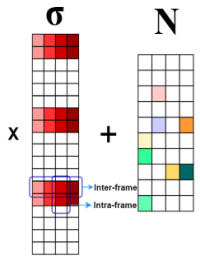

Fig. 1. Schematic illustration of MFC-BSBL that exploits both inter-frame correlation and intra-frame correlation of the conductivity distribution. (a) Multi-frame conductivity images are vectorized and arranged in columns. (b) Blocks of inter-frame correlation and intra-frame correlation.

In this section, we introduce the MFC-BSBL method based on the SBL. SBL was initially proposed for data regression and data classification and was then applied to sparse signal recovery [22], [23].The idea is to use the Bayesian rule to find 
the posterior probability of $p(\sigma \mid \mathbf{V} ; \Theta)$, where $\Theta$ denotes the set of all parameters. Given $\Theta, \sigma$ can be calculated using the MAP estimate[15].

An important characteristic of EIT technology is its high time resolution. Therefore, when the impedance distribution of the object changes, multi-frame data of boundary potentials can be quickly obtained in a short time. Because the boundary data are acquired continuously, for any piece of the obtained data, the corresponding reconstructed images should have a similar impedance distribution. Based on this similarity prior, combined with the structure-aware priors [15], [16], we proposed the MFC-BSBL algorithm to solve the MAP estimate. By exploring and exploiting the sparsity, interframe correlation, and intra-frame correlation of impedance distribution, the MFC-BSBL algorithm can effectively reduce the artifacts in reconstructed images and improve the accuracy of tactile sensors for contact detection.

The imaging result of the tactile sensor is relative to the contact region because the change in conductivity is affected by the contact position. In Figure 1, multi-frame conductivity images are vectorized and arranged in columns to form the matrix $\sigma$, where each row denotes a pixel in the image. We divide the images into many blocks: when the impedance changes, we can find that most of the blocks remain unchanged and only a small part of the blocks will change. In this paper, we consider a general case where block partitions overlap each other and have an equal size of $h$. Accordingly, $g=N-h+1$ overlapped blocks are defined at frame $l$ and $\sigma_{:, l}=\left[\Psi_{:,[1]}, \ldots, \Psi_{:,[g]}\right] \mathbf{X}_{:, l}$, where $\sigma_{:, l}$ is the $l$-th column of the matrix $\sigma$.

$$
\begin{gathered}
\Psi_{:,[j]}=\left[\mathbf{0}_{(j-1) \times h}^{\top}, \mathbf{I}_{h \times h}^{\top}, \mathbf{0}_{(N-j-h+1) \times h}^{\top}\right]^{\top} \in \mathbb{R}^{N \times h} \\
\mathbf{X}_{[i], l}=\left[\mathbf{X}_{i, l}, \ldots, \mathbf{X}_{i+h-1, l}\right]^{\top} \in \mathbb{R}^{h \times 1} \\
\mathbf{X}_{:, l}=\left[\mathbf{X}_{[1], l}^{\top}, \ldots, \mathbf{X}_{[g], l}^{\top}\right]^{\top} \in \mathbb{R}^{g h \times 1}
\end{gathered}
$$

where $\mathbf{X}_{[i], l}$ is the $i$-th block in the $l$ frame, and $\mathbf{X}_{:, l}$ denotes all blocks in the $l$ frame. Model (8) can be rewritten as

$$
\mathbf{V}=\mathbf{J} \sigma+\mathbf{N}=\mathbf{J} \Psi \mathbf{X}+\mathbf{N}=\Phi \mathbf{X}+\mathbf{N}
$$

where $\mathbf{V} \in \mathbb{R}^{M \times L}, \Phi \in \mathbb{R}^{M \times g h}, \mathbf{X} \in \mathbb{R}^{g h \times N}, \mathbf{N} \in$ $\mathbb{R}^{M \times L}$. Note that when blocks have no overlap $(h=1)$, the model becomes $\mathbf{V} \in \mathbb{R}^{M \times L}, \Phi \in \mathbb{R}^{M \times N}, \mathbf{X} \in \mathbb{R}^{N \times N}, \mathbf{N} \in$ $\mathbb{R}^{M \times L}$.

To facilitate the development of this algorithm, we assume that the $i$-th block $\mathbf{X}_{[i], \text {, }}$ has a parameterized Gaussian distribution $p\left(\operatorname{vec}\left(\mathbf{X}_{[i],:}^{\top}\right) ; \gamma_{i}, \mathbf{B}, \mathbf{A}_{i}\right)=\mathcal{N}\left(0,\left(\gamma_{i} \mathbf{A}_{i}\right) \otimes \mathbf{B}\right)$, $\mathbf{B} \in \mathbb{R}^{L \times L}, \mathbf{A}_{i} \in \mathbb{R}^{h \times h}$, where $\mathbf{B}$ and $\mathbf{A}_{i}$ are unknown positive definite matrices capturing the intra-frame correlation structure in each row of $\mathbf{X}_{[i]}$,: and the inter-frame correlation structure in each column of $\mathbf{X}_{[i], \text {,: }}$, and $\otimes$ is the Kronecker product operator. The parameter $\gamma_{i}$ is used to control the structured sparsity of $i$-th block. Because only a small part of the blocks are non-sparse, most of $\gamma_{i}(1,2, \ldots, g)$ are zero.

$$
p\left(\operatorname{vec}\left(\mathbf{X}^{\top}\right) ; B,\left\{\gamma_{i}, \mathbf{A}_{i}\right\}_{i}\right)=\mathcal{N}(0, \Pi \otimes \mathbf{B})
$$

where $\Pi$ is a block diagonal matrix

$$
\Pi=\left[\begin{array}{llll}
\gamma_{1} \mathbf{A}_{1} & & & \\
& \gamma_{2} \mathbf{A}_{2} & & \\
& & \ddots & \\
& & & \gamma_{g} \mathbf{A}_{g}
\end{array}\right]
$$

Similar to [22], we assume that the noise matrix $\mathbf{N}$ have the Gaussian distribution and the rows are mutually independent, we can get

$$
p\left(\operatorname{vec}\left(\mathbf{N}^{\top}\right) ; \lambda, \mathbf{B}\right)=\mathcal{N}(0, \lambda \mathbf{I} \otimes \mathbf{B})
$$

Accordingly, let $\mathbf{x}=\operatorname{vec}\left(\mathrm{X}^{\top}\right), \mathbf{u}=\operatorname{vec}\left(\mathbf{V}^{\top}\right), \mathbf{v}=\operatorname{vec}\left(\mathbf{N}^{\top}\right)$, we can obtain the posterior of $\mathbf{x}$ given $\mathbf{u}$ as:

$$
p(\mathbf{x} \mid \mathbf{u} ; \Theta)=\mathcal{N}\left(\mu_{0}, \Sigma_{0}\right)
$$

where $\Theta$ denotes the hyper-parameters $\left\{\gamma_{i}, \mathbf{A}_{i}\right\}_{i=1}^{g}, \lambda, \mathbf{B}$. The mean $\mu_{0}$ and covariance matrix $\Sigma_{0}$ are given by

$$
\begin{gathered}
\Sigma_{0}=\left(\Pi-\Pi \Phi^{\top}\left(\lambda \mathbf{I}+\Phi \Pi \Phi^{\top}\right)^{-1} \Phi \Pi\right) \otimes \mathbf{B} \\
\mu_{0}=\operatorname{vec}\left(\mathbf{V}^{\top}\left(\lambda \mathbf{I}+\Phi \Pi \Phi^{\top}\right)^{-1} \Phi \Pi\right)
\end{gathered}
$$

Once we obtain the hyper-parameters, the MAP estimate of $\mathrm{X}$ can be calculated using the posterior mean

$$
\mathbf{X} \leftarrow \Pi \Phi^{\top}\left(\lambda \mathbf{I}+\Phi \Pi \Phi^{\top}\right)^{-1} \mathbf{V}
$$

To estimate the hyper-parameters, we use the Type II maximum likelihood method [26] and obtain the cost function:

$$
\begin{aligned}
\mathcal{L}(\Theta) & =-2 \log \int p(\mathbf{u} \mid \mathbf{x} ; \lambda) p\left(\mathbf{x} ;\left\{\gamma_{i}, \mathbf{A}_{i}\right\}, \mathbf{B}\right) d x \\
& =\mathbf{u}^{\top} \Sigma_{\mathbf{u}}{ }^{-1} \mathbf{u}+\log \left|\Sigma_{\mathbf{u}}\right|
\end{aligned}
$$

where $\Sigma_{\mathbf{u}}=\lambda \mathbf{I} \otimes \mathbf{B}+\Phi(\Pi \otimes \mathbf{B}) \Phi^{\top}$. However, as shown in model (14), there is a coupling between $\mathbf{A}_{i}$ and $\mathbf{B}$ when we directly estimate the hyperparameters, which will cause complex computation. Thus, an alternating-learning method was adapted to estimate the parameters $\left\{\gamma_{i}, \mathbf{A}_{i}\right\}_{i=1}^{g}, \lambda$ and $B$ in turn. First, assuming that $\mathbf{B}$ has been estimated, and letting $\tilde{\mathbf{V}}=\mathbf{V} \mathbf{B}^{(-1 / 2)}, \tilde{\mathbf{X}}=\mathbf{X} \mathbf{B}^{(-1 / 2)}, \tilde{\mathbf{N}}=\mathbf{N B} \mathbf{B}^{(-1 / 2)}$. Then, we can obtain $\tilde{\mathbf{Y}}=\Phi \tilde{\mathbf{X}}+\tilde{\mathbf{V}}$ with $p(\tilde{\mathbf{X}} ; \Pi)=\prod_{i=1}^{L} p\left(\tilde{\mathbf{X}}_{:, i}\right) \sim$ $\prod_{i} \mathcal{N}(0, \Pi)$ and $p(\tilde{\mathbf{N}} ; \lambda)=\prod_{i=1}^{L} p\left(\tilde{\mathbf{N}}_{:, i}\right) \sim \prod_{i} \mathcal{N}(0, \lambda \mathbf{I})$.

According to the ARD and Woodbury inversion identity, we can obtain the likelihood $p\left(\tilde{\mathbf{V}}_{:, i} \mid \tilde{\mathbf{X}}_{:, i} ; \lambda\right)=\mathcal{N}\left(\Phi \tilde{\mathbf{X}}_{:, i}, \lambda \mathbf{I}\right)$ and the posterior $p\left(\tilde{\mathbf{X}}_{:, i} \mid \tilde{\mathbf{V}}_{:, i} ; \lambda, \Pi\right)=\mathcal{N}\left(\mu_{:, i}, \Sigma\right)$ with the mean $\mu_{:, i}$ and the covariance matrix $\Sigma$

$$
\begin{gathered}
\mu_{:, i}=\Pi \Phi^{\top}\left(\lambda \mathbf{I}+\Phi \Pi \Phi^{\top}\right)^{-1} \tilde{\mathbf{V}}_{:, i} \\
\Sigma=\Pi-\Pi \Phi^{\top}\left(\lambda \mathbf{I}+\Phi \Pi \Phi^{\top}\right)^{-1} \Phi \Pi
\end{gathered}
$$

Then, similar to (21), the solution can be calculated as

$$
\tilde{\mathbf{X}} \leftarrow \Pi \Phi^{\top}\left(\lambda \mathbf{I}+\Phi \Pi \Phi^{\top}\right)^{-1} \tilde{\mathbf{V}}, \mathbf{X} \leftarrow \tilde{\mathbf{X}} \mathbf{B}^{(-1 / 2)}
$$


1) Estimate the parameters $\left\{\gamma_{i}, \mathbf{A}_{i}\right\}_{i=1}^{g}, \lambda$ : Using the expectation maximization (EM) method in [22], we can get the Q-function for estimating

$$
\begin{aligned}
Q(\Pi) & =E_{\tilde{\mathbf{X}} \mid \tilde{\mathbf{V}} ; \Theta^{\text {old }}}\left[\log p\left(\tilde{\mathbf{X}} ;\left\{\gamma_{i}\right\}_{i},\left\{\mathbf{A}_{i}\right\}_{i}\right)\right] \\
& =-\frac{L}{2} \sum_{i=1}^{g} h \log \gamma_{i}-\frac{L}{2} \sum_{i=1}^{g} \log \left|\mathbf{A}_{i}\right| \\
& -\frac{1}{2} \sum_{l=1}^{L} \sum_{j=1}^{g} \operatorname{Tr}\left[\left(\gamma_{j} \mathbf{A}_{j}^{-1}\right)^{-1}\left(\Sigma_{[j]}+\mu_{[j], l} \mu_{[j], l}^{\top}\right)\right]
\end{aligned}
$$

where $\Sigma_{[j]}$ is the $j$-th diagonal block in $\Sigma, \mu_{[j], l}$ is the $j$-th block in the l-th column of $\mu$, and $\Theta^{\text {old }}$ denotes the hyperparameters of previous iteration. We can get the updating rule for $\gamma_{i}$ and $\mathbf{A}_{i}$ by setting to zero the derivative of (26) with respect to $\gamma_{i}$ and $\mathbf{A}_{i}$ :

$$
\begin{gathered}
\mathbf{A}_{i} \leftarrow \frac{1}{L} \sum_{l=1}^{L} \frac{\left(\Sigma_{[i]}+\mu_{[i], l} \mu_{[i], l}^{\top}\right)}{\gamma_{i}} \\
\gamma_{i} \leftarrow \frac{1}{L h} \sum_{l=1}^{L} \operatorname{Tr}\left[\mathbf{A}^{-1}\left(\Sigma_{[i]}+\mu_{[i], l} \mu_{[i], l}^{\top}\right)\right]
\end{gathered}
$$

Similarly, we can write the Q-function and derive the updating rule for $\lambda$

$$
\begin{aligned}
Q(\lambda)= & E_{\tilde{\mathbf{X}} \mid \tilde{\mathbf{V}} ; \Theta^{\text {old }}}[\log P(\tilde{\mathbf{V}} \mid \tilde{\mathbf{X}} ; \lambda)] \\
= & -\frac{N L}{2} \log \lambda-\frac{1}{2 \lambda}\|\tilde{\mathbf{V}}-\Phi \mu\|_{\mathcal{F}}^{2}-\frac{1}{2 \lambda} T_{r}\left(\Sigma \Phi^{\top} \Phi\right) \\
& \lambda \leftarrow \frac{1}{N L}\|\tilde{\mathbf{V}}-\Phi \mu\|_{F}^{2}+\frac{1}{N} T_{r}\left(\Sigma \Phi^{\top} \Phi\right)
\end{aligned}
$$

To improve the robustness at low signal-to-noise (SNR) situations, the above rule is modified as:

$$
\lambda \leftarrow \frac{1}{N L}\|\tilde{\mathbf{V}}-\Phi \mu\|_{F}^{2}+\frac{1}{N} \sum_{i=1}^{g} T_{r}\left(\Sigma_{[i]} \Phi_{:,[i]}^{\top} \Phi_{:,[i]}\right)
$$

where $\Phi_{:,[i]}$ denotes the consecutive columns in which correspond to the $i$-th block in $\tilde{\mathbf{V}}$. The updating rules for $\left\{\gamma_{i}, \mathbf{A}_{i}\right\}_{i=1}^{g}, \lambda$ have satisfied the reconstruction performance, but are slow because calculating $\gamma_{i}$ is time-consuming. Here, we adapt the bound-optimization method in [24] to obtain a faster updating rule for $\gamma_{i}$. By finding an upper bound for the second term in (22), and then calculating the minimum of the cost function, we can obtain

$$
\gamma_{i} \leftarrow \sqrt{\frac{\operatorname{Tr}\left(\mathbf{X}_{[i],:} \mathbf{B}^{-1} \mathbf{X}_{[i],:}^{\top} \mathbf{A}_{i}^{-1}\right) / L}{\operatorname{Tr}\left(\left(\lambda \mathbf{I}+\Phi \Pi \Phi^{\top}\right)^{-1} \Phi_{:,[i]} \mathbf{A}_{i} \Phi_{:,[i]}^{\top}\right)}}
$$

2) Estimate the parameters $\mathbf{B}$ : Once hyper-parameters $\left\{\gamma_{i}, \mathbf{A}_{i}\right\}_{i=1}^{g}, \lambda$ are estimated, the updating rule for $\mathbf{B}$ can be derived by calculating the gradient of the cost function (22) with respect to $\mathbf{B}$ :

$$
\tilde{\mathbf{B}} \leftarrow \sum_{i=1}^{g} \gamma_{i}^{-1} \tilde{\mathbf{X}}_{[i],:}^{\top} \tilde{\mathbf{X}}_{[i],:}=\sum_{i=1}^{g} \gamma_{i}^{-1} \mathbf{X}_{[i],:}^{\top} \mathbf{A}_{i}^{-1} \mathbf{X}_{[i],:}
$$

$$
\mathbf{B} \leftarrow \frac{\tilde{\mathbf{B}}}{\|\tilde{\mathbf{B}}\|_{\mathcal{F}}}
$$

where (34) removes the ambiguity between $\mathbf{B}$ and $\gamma_{i}$. This updating rule can perform well in noiseless cases but is not robust [24] at low SNR due to errors from the estimated $\gamma_{i}$ and $\mathbf{X}_{[i],:}$.To solve this problem, a regularization method is adopted to constrain the estimates of $\mathbf{B}$ and $\mathbf{A}_{\mathbf{i}}$.

Accordingly, the estimate $\tilde{\mathbf{B}}$ can be regularized by:

$$
\tilde{\mathbf{B}} \leftarrow \sum_{i=1}^{g} \frac{\mathbf{X}_{[i],:}^{\top} \mathbf{A}_{i}^{-1} \mathbf{X}_{[i],:}}{\gamma_{i}}+\eta \mathbf{I}
$$

where $\eta$ is a positive constant. This regularization eliminates the influence of noise covariance and improves the stability of the algorithm. To estimate $\mathrm{A}_{i}$, we adapted the Toeplitz structure to regularize the covariance structure. A $h \times h$ Toeplitz matrix of the form is given by

$$
\begin{gathered}
\tilde{\mathbf{A}}_{\mathbf{i}} \leftarrow\left[\begin{array}{cccc}
1 & r \cdots & r^{h-1} \\
\vdots & \vdots & \vdots \\
r^{h-1} & r^{h-2} & \cdots & 1
\end{array}\right] \\
\mathbf{A}_{i} \leftarrow \frac{\tilde{\mathbf{A}}_{\mathbf{i}}}{\left\|\tilde{\mathbf{A}}_{\mathbf{i}}\right\|_{\mathcal{F}}}
\end{gathered}
$$

where $r \leftarrow(1 / g) \sum_{i=1}^{g} r_{i}$ is the Auto-Regressive(AR) coefficient and $r_{i}$ can be calculated by

$$
r_{i} \leftarrow \operatorname{sign}\left(m_{1}^{i} / m_{0}^{i}\right) \min \left\{\left|\left(m_{1}^{i} / m_{0}^{i}\right)\right|, 0.9\right\}
$$

where $m_{0}^{i}$ is the average of entries in the main diagonal of $\mathbf{A}_{i}$ and $m_{1}^{i}$ is the average of entries in the main sub-diagonal of $\mathbf{A}_{i}$.

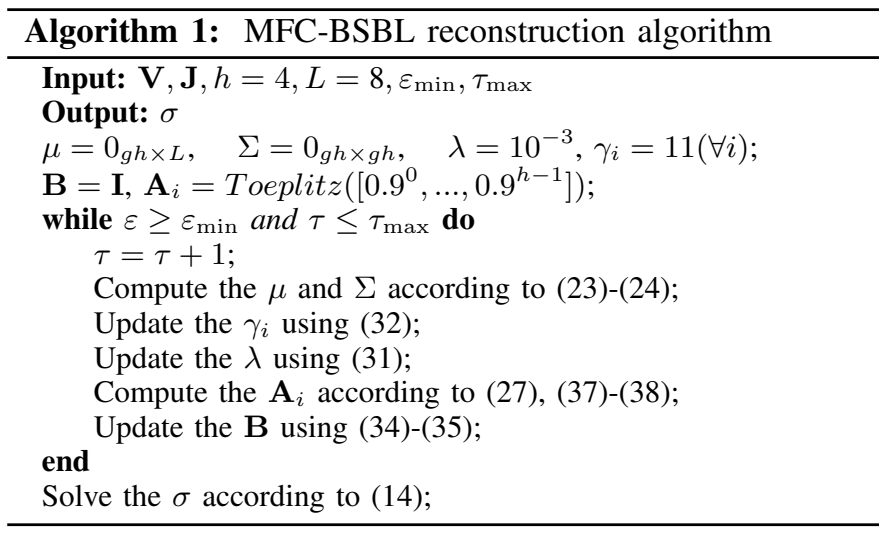

To date, all updating rules for hyper-parameters have been derived and the flow chart of MFC-BSBL is shown in Algorithm 1. In addition, two criteria were used to stop the algorithm. One is that the maximum change in any entry of the estimated in successive iterations is smaller than $10^{-5}$. The second criterion is that the iteration number is larger than 50 , as the result has stabilized at this time. 


\section{NUMERICAL SIMULATIONS}

\section{A. Numerical model}

To verify the contact detection effectiveness of the tactile sensor using MFC-BSBL, we conducted numerical simulations using EIDORS [25]. This software is an open-source EIT tool in MATLAB libraries that can be employed to create FEM models, solve EIT forward problems, reconstruct images, etc.

We used the EIDORS to build the tactile sensor model and mesh the detective part of the tactile sensor into $56 \times 56 \times 2$ triangular elements. Accordingly, the number of pixels in the reconstructed images for this experiment is $N=6272$. By setting the side length of the detective part to 1 , we can limit the coordinate of the contact position to 0 to 1 , which is equivalent to performing a normalization that can facilitate subsequent calculations. The setting of the frame length depends on the data acquisition rate of the tactile sensor. Currently, the tactile sensor will take approximately one second to collect eight frame data. Therefore, we set the frame length $L$ to 8 . As for the other settings, we default on EIDORS.

\section{B. Evaluation criteria}

To evaluate the performance of the proposed MFC-BSBL algorithm and the tactile sensor system, we introduce two criteria: position error (PE) and mean intersection over union (MIOU). PE is used to illustrate the detection accuracy of the contact position of the tactile sensor, which can be defined as:

$$
\mathrm{PE}=\frac{1}{S} \sum_{i=1}^{S}\left|R m_{i}-R t_{i}\right|
$$

where $S$ is the number of regions of impedance change. $R m_{i}$ is the centroid of the $i$-th impedance change region of the real impedance distribution, and $R t_{i}$ is the centroid of the $i$-th impedance change region of the reconstructed impedance distribution. The smaller the position error obtained by the reconstruction algorithm, the smaller is the PE value.

MIOU is used to measure the difference between the regions of the real impedance distribution and the reconstructed impedance distribution, which is defined as

$$
\begin{aligned}
\mathrm{MIOU} & =\frac{1}{S} \sum_{i=1}^{S} \frac{T P}{(T P+F N)+(T P+F P)-T P} \\
& =\frac{1}{S} \sum_{i=1}^{S} \frac{T P}{T P+F N+F P}
\end{aligned}
$$

where TP denotes the overlapped region of the real impedance change and reconstructed impedance, $(\mathrm{TP}+\mathrm{FN})$ denotes the real impedance change of the contact region and $(\mathrm{TP}+\mathrm{FP})$ denotes the reconstructed distribution of the contact region. In Figure 2, we draw a schematic diagram of the MIOU criterion when $S=1$. As we can see, a larger MIOU indicates that the reconstructed conductivity change region is closer to the real contact region, which demonstrates better performance of the reconstruction algorithm.

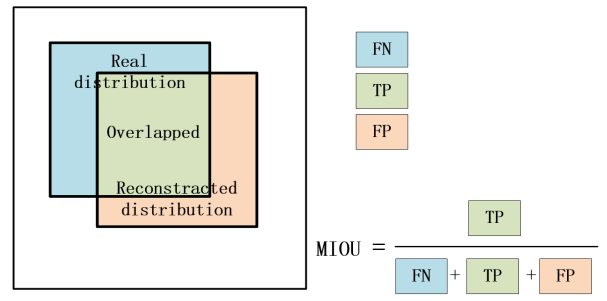

Fig. 2. Schematic diagram of the MIOU criterion. The outermost square denotes the conductive plate. The two squares inside refer to the real impedance change $(\mathrm{TP}+\mathrm{FN})$ of the contact region and the reconstructed distribution $(\mathrm{TP}+\mathrm{FP})$ of the contact region respectively.

\section{Results}

We compared three reconstruction algorithms with the proposed method and displayed the simulation results of various contact cases, as shown in Figure 3. As can be seen in the first column, brown objects represent the corresponding regions and contact positions of the impedance change in the detective part. Our goal is to reconstruct the exact location and region of impedance change when given boundary measurements. We listed the real region of impedance change and compared it with reconstructed images reconstructed by TV, NOSER, SA-SBL, and MFC-BSBL algorithms. It can be observed that SA-SBL and MFC-BSBL algorithms can effectively remove artifacts and noise in reconstructed images, especially for small regions of impedance change. In particular, we listed the real contact position of the impedance change in each column of Figure 3 and marked it with a red dot. Although the four algorithms can achieve effective contact position detection, the MFC-BSBL algorithm achieves the closest result to the real position. To quantitatively evaluate the performance of the

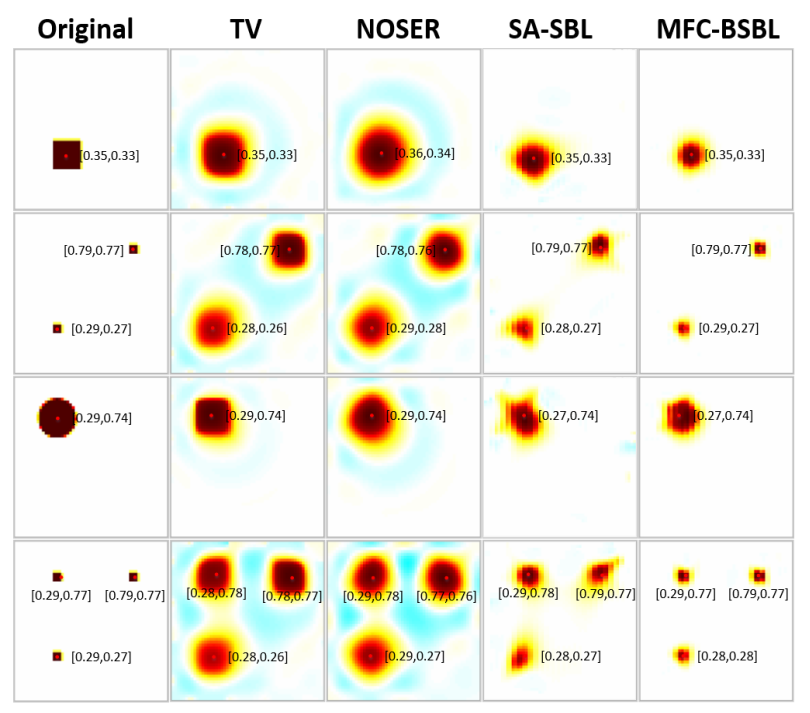

Fig. 3. Numerical simulations of four contact situations (one-target, multitarget, square target and round target).

algorithms, we adopted the two commonly used evaluation criteria mentioned above and conducted 1000 Monte Carlo trials with input SNRs ranging from $20 \mathrm{~dB}$ to $70 \mathrm{~dB}$. The results are shown in Figure 4. 


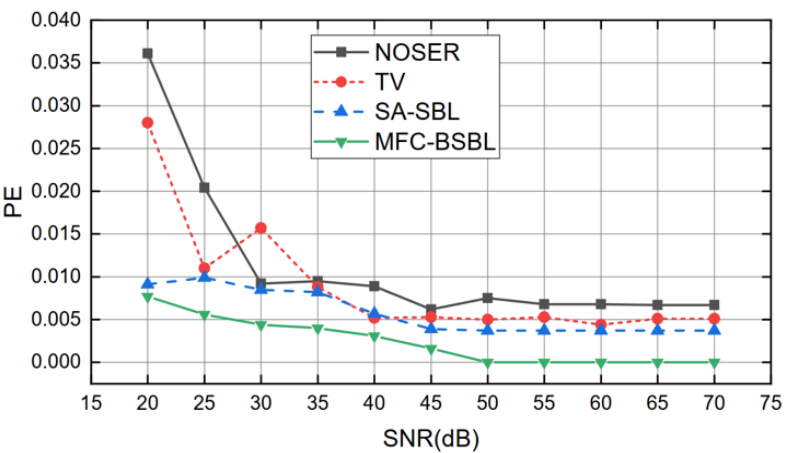

(a)

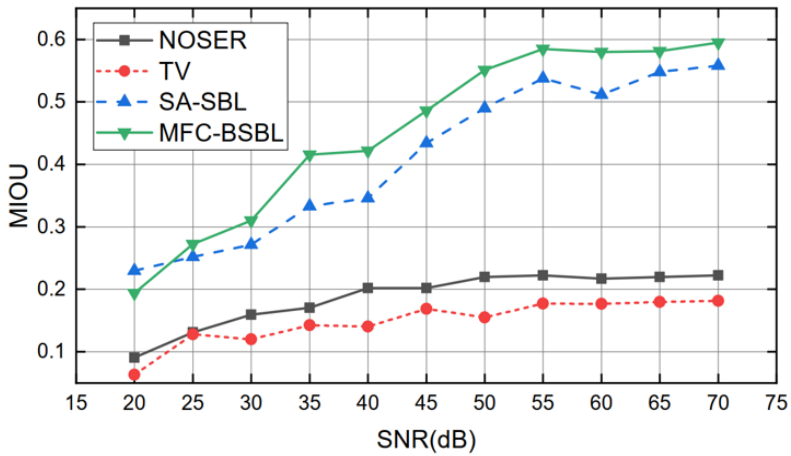

(b)

Fig. 4. Reconstruction performance of four algorithms in different noise levels.

It can be observed that as the SNR increases, the MFCBSBL can obtain a better reconstruction performance than the other three methods. When the SNR approaches $50 \mathrm{~dB}$, the results no longer change. In comparison, traditional algorithms yield a larger PE and a smaller MIOU, indicating worse results. On the other hand, we can see that even though the environment is noisy, the proposed method can still achieve accurate contact detection, which demonstrates its robustness.

\section{EXPERIMENTAL STUDY}

In this section, we first describe our tactile sensor system in detail and evaluate the proposed MFC-BSBL method using real-collected data. Then, three reconstruction methods and two evaluation criteria were compared to demonstrate the superiority of the MFC-BSBL method. Finally, we introduce and discuss the imaging results of the contact detection using our tactile sensor system.

\section{A. Implementation of data acquisition board}

A data acquisition board and circuit schematic are shown in Figure 5. The portable EIT system is based on the AD5940 analog chip, which is a high-precision and lowpower analog front end that can be applied to electrochemicalbased measurement applications [26]. It consists of two highprecision excitation loops and one common measurement channel, which can achieve a wide measurement capability of the sensor under test. We adopt a 4-wire bio-impedance analysis (BIA) approach to measure the impedance change of the tactile sensor. As shown in Figure 5(top), a known voltage is excited to the tactile sensor through a high-precision $\mathrm{AC}$ voltage source. Simultaneously, a common-mode voltage is applied across the sensor. To calculate the impedance data,

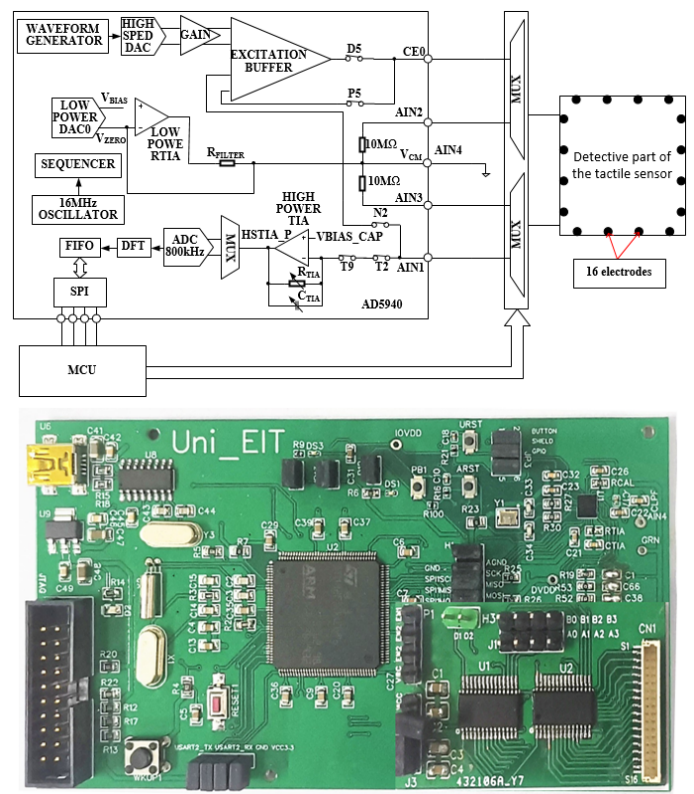

Fig. 5. The data acquisition board and circuit schematic

a high-speed transimpedance amplifier (TIA) was adapted to convert the current to a voltage to be read by a 16-bit analogto-digital converter (ADC). Simultaneously, a discrete Fourier transform (DFT) is performed on the ADC results, and the real and imaginary parts are stored in the data FIFO, which can be read by the host microcontroller in the EIT system.

Our goal is to image the impedance distribution when the detective part of the tactile sensor is in contact. Therefore, the detective part, as a perception unit that interacts with the outside world, should be soft and adaptable to various geometries. The commonly detected parts can be developed by various solid, fabric, pliable, and paintable conductors. For practicality, we select the paintable conductors (GRAPHITE128, Germany), which has a surface resistance of $1 \mathrm{~K}$ to $2 \mathrm{~K}$.

In this experiment, we sprayed the conductive paint evenly on a square A4 paper to obtain a soft conductive plate. Then we evenly placed 16 electrodes around the plate and connected these electrodes to the data acquisition part of the tactile sensor. Once the tactile sensor detects the external contact, it immediately displays the contact position and region on the laptop.

\section{B. Contact detection experiment}

In this section, real contact detection experiments are designed to further validate the performance of the tactile sensor system and the proposed MFC-BSBL EIT reconstruction algorithm.

As can be seen in the first column of Figure 6, a conductive square plate with a side length of $20 \mathrm{~cm}$ is shown. Sixteen stainless steel electrodes were placed around the plate, each with a contact area of $7 \mathrm{~mm}^{2}$. Square and round conductive blocks were placed on the detective part of the tactile 
sensor, and the corresponding boundary measurement data were recorded. We conducted various contact experiments and compared the reconstruction performance of the four algorithms. Four representative results are shown in Figure 6. We displayed the impedance change region of the reconstructed images and the contact position of the reconstructed images, and marked the real impedance change region with a black square or circle. The first two rows of Figure 6 describe the

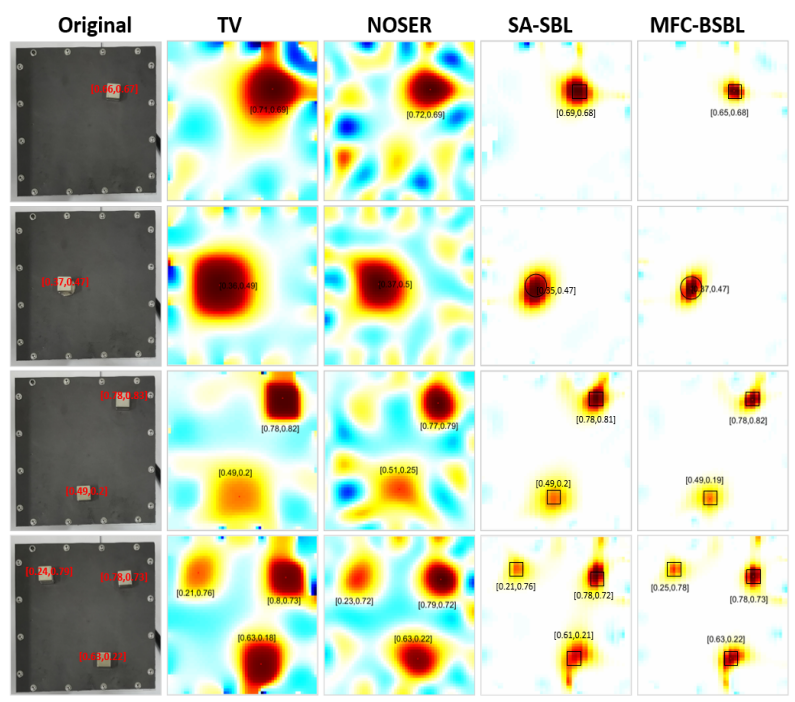

Fig. 6. The results of contact detection experiments. (The black square box denotes the real touch region, and the red label denote the real touch position)

one-target contact detection results of two different shapes including round and square conductive blocks. As we can see that SA-SBL and MFC-BSBL algorithms can greatly reduce the interference of noise and remove artifacts in the reconstructed images. Compared with SA-SBL, the reconstructed impedance change region using MFC-BSBL is closer to the marked object, which indicates that the tactile sensor using the proposed algorithm can achieve a better contact detection for the one-target impedance change region. Additionally, by comparing the detection results of the contact position, we can see that the proposed algorithm achieves the closest result to the real value, which indicates that the proposed tactile sensor using the proposed algorithm can achieve accurate one-target contact position detection. The last two rows of Figure 6 illustrate two multi-target contact detection results including two and three objects. From the comparison of reconstructed images, we can see that the proposed tactile sensor using the MFC-BSBL algorithm can obtain the best reconstruction results. On the one hand, comparing the marked real impedance change region with the reconstructed images, we cfound that MFC-BSBL achieved the closest result. On the other hand, by calculating the detection error of multi-target contact position, we can see that the proposed method can achieve the most accurate detection. These results show that the tactile sensor using the proposed algorithm can effectively realize multi-target contact detection.

To compare the experimental results more intuitively, we averaged the PE and MIOU values of the four typical contact detection cases and displayed the results in Figure 7. We found that the proposed algorithm obtained the largest MIOU and the smallest PE, which demonstrated that the proposed tactile sensor system using the MFC-BSBL method can accurately detect the contact position and contact region.

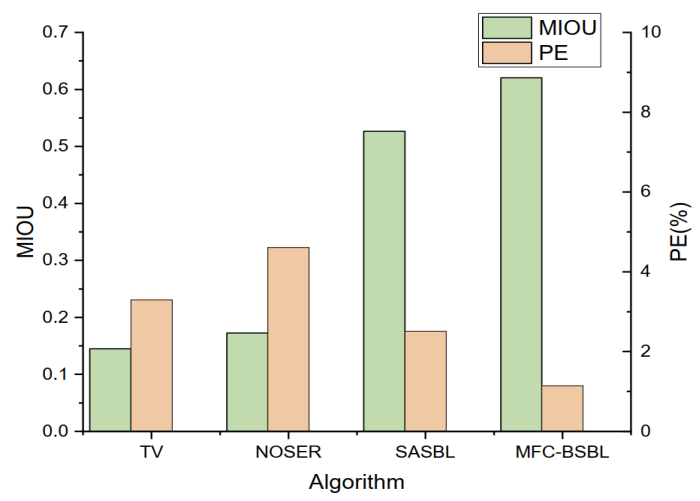

Fig. 7. Performance comparison of four reconstruction algorithms in contact detection experiment

\section{CONCLUSION}

We proposed a multi-frame constrained block sparse Bayesian learning method to explore the sparsity, intra-frame correlation and inter-frame correlation of impedance distribution, and designed a flexible tactile sensing system to achieve accurate EIT reconstruction of contact detection. Both numerical simulations and contact detection experiments were conducted to evaluate the proposed method for different cases to identify one, multiple, round, and square targets, respectively. Compared with the TV, NOSER, and SA-SBL methods, the proposed MFC-BSBL algorithm for image reconstruction can achieve better performance in removing artifacts and noise interference for EIT-based tactile sensing.

\section{REFERENCES}

[1] F. Visentin and P. Fiorini, "A flexible sensor for soft-bodied robots based on electrical impedance tomography," in 2018 IEEE International Conference on Soft Robotics (RoboSoft). IEEE, 2018, pp. 158-163.

[2] S. Russo, R. Assaf, N. Carbonaro, and A. Tognetti, "Touch position detection in electrical tomography tactile sensors through quadratic classifier," IEEE Sensors Journal, vol. 19, no. 2, pp. 474-483, 2018.

[3] Y. Yang, W. Zhou, X. Chen, J. Ye, and H. Wu, "A flexible touching sensor with the variation of electrical impedance distribution," Measurement, p. 109778, 2021.

[4] A. Nagakubo, H. Alirezaei, and Y. Kuniyoshi, "A deformable and deformation sensitive tactile distribution sensor," in 2007 IEEE International Conference on Robotics and Biomimetics (ROBIO). IEEE, 2007, pp. 1301-1308

[5] Z. Wei, D. Liu, and X. Chen, "Dominant-current deep learning scheme for electrical impedance tomography," IEEE Transactions on Biomedical Engineering, vol. 66, no. 9, pp. 2546-2555, 2019.

[6] A. Adler, J. H. Arnold, R. Bayford, A. Borsic, B. Brown, P. Dixon, T. J. Faes, I. Frerichs, H. Gagnon, Y. Gärber et al., "Greit: a unified approach to $2 \mathrm{~d}$ linear eit reconstruction of lung images," Physiological measurement, vol. 30, no. 6, p. S35, 2009.

[7] M. Cheney, D. Isaacson, J. C. Newell, S. Simske, and J. Goble, "Noser: An algorithm for solving the inverse conductivity problem," International Journal of Imaging systems and technology, vol. 2, no. 2, pp. 66-75, 1990.

[8] A. Borsic, B. M. Graham, A. Adler, and W. R. Lionheart, "In vivo impedance imaging with total variation regularization," IEEE transactions on medical imaging, vol. 29, no. 1, pp. 44-54, 2009. 
[9] B. Sun, S. Yue, Z. Hao, Z. Cui, and H. Wang, "An improved tikhonov regularization method for lung cancer monitoring using electrical impedance tomography," IEEE Sensors Journal, vol. 19, no. 8, pp. 3049 $3057,2019$.

[10] S. J. Hamilton, A. Hänninen, A. Hauptmann, and V. Kolehmainen, "Beltrami-net: domain-independent deep d-bar learning for absolute imaging with electrical impedance tomography (a-eit)," Physiological measurement, vol. 40, no. 7, p. 074002, 2019.

[11] D. Liu, D. Smyl, D. Gu, and J. Du, "Shape-driven difference electrical impedance tomography," IEEE Transactions on Medical Imaging, vol. 39, no. 12, pp. 3801-3812, 2020.

[12] H. Park, K. Park, S. Mo, and J. Kim, "Deep neural network based electrical impedance tomographic sensing methodology for large-area robotic tactile sensing," IEEE Transactions on Robotics, 2021.

[13] Y. Zhang, Z. Lin, X. Huang, X. You, J. Ye, and H. Wu, "A large-area stretchable, textile-based tactile sensor," Advanced Materials Technologies, vol. 5, no. 4, p. 1901060, 2020.

[14] Z. Wei, Z. Zong, and Y. Wang, "A reliable deep learning scheme for nonlinear reconstructions in electrical impedance tomography," IEEE Transactions on Computational Imaging, vol. 7, pp. 789-798, 2021.

[15] Z. Zhang and B. D. Rao, "Sparse signal recovery with temporally correlated source vectors using sparse bayesian learning," IEEE Journal of Selected Topics in Signal Processing, vol. 5, no. 5, pp. 912-926, 2011.

[16] S. Liu, J. Jia, Y. D. Zhang, and Y. Yang, "Image reconstruction in electrical impedance tomography based on structure-aware sparse bayesian learning," IEEE transactions on medical imaging, vol. 37, no. 9, pp. 2090-2102, 2018.

[17] S. Liu, H. Wu, Y. Huang, Y. Yang, and J. Jia, "Accelerated structure-aware sparse bayesian learning for three-dimensional electrical impedance tomography," IEEE Transactions on Industrial Informatics, vol. 15 , no. 9, pp. 5033-5041, 2019.

[18] J. Xiang, Y. Dong, and Y. Yang, "Multi-frequency electromagnetic tomography for acute stroke detection using frequency constrained sparse bayesian learning," arXiv preprint arXiv:2003.00998, 2020.

[19] L. Yin, K. Wang, T. Tong, Y. An, H. Meng, X. Yang, and J. Tian, "Improved block sparse bayesian learning method using k-nearest neighbor strategy for accurate tumor morphology reconstruction in bioluminescence tomography," IEEE Transactions on Biomedical Engineering, 2019.

[20] K.-S. Cheng, D. Isaacson, J. Newell, and D. G. Gisser, "Electrode models for electric current computed tomography," IEEE Transactions on Biomedical Engineering, vol. 36, no. 9, pp. 918-924, 1989.

[21] P. J. Vauhkonen, M. Vauhkonen, T. Savolainen, and J. P. Kaipio, "Three-dimensional electrical impedance tomography based on the complete electrode model," IEEE Transactions on Biomedical Engineering, vol. 46, no. 9, pp. 1150-1160, 1999.

[22] M. E. Tipping, "Sparse bayesian learning and the relevance vector machine," Journal of machine learning research, vol. 1, no. Jun, pp. 211-244, 2001.

[23] D. P. Wipf and B. D. Rao, "Sparse bayesian learning for basis selection," IEEE Transactions on Signal processing, vol. 52, no. 8, pp. 2153-2164, 2004.

[24] Z. Zhang, T.-P. Jung, S. Makeig, and B. D. Rao, "Compressed sensing for energy-efficient wireless telemonitoring of noninvasive fetal ecg via block sparse bayesian learning," IEEE Transactions on Biomedical Engineering, vol. 60, no. 2, pp. 300-309, 2012.

[25] A. Adler and W. R. Lionheart, "Uses and abuses of eidors: an extensible software base for eit," Physiological measurement, vol. 27, no. 5, p. S25, 2006.

[26] A. Devices, "High precision, impedance, and electrochemical front end ad5940/5941," Data Sheet. [Online]. Available: https://www.analog. com/media/en/technical-documentation/data-sheets/AD5940-5941.pdf 